\title{
A multi-criteria vehicle-target allocation assessment model for network-centric Joint Air Operations
}

\author{
Madjid Tavana* \\ Management Information Systems, \\ Lindback Distinguished Chair of Information Systems, \\ La Salle University, \\ Philadelphia, PA 19141, USA \\ E-mail: tavana@lasalle.edu \\ *Corresponding author

\section{Matthew D. Bailey} \\ Management Department, \\ Bucknell University, \\ Lewisburg, PA 17837, USA \\ E-mail: mdb025@bucknell.edu
}

\section{Timothy E. Busch}

Air Force Research Laboratory, AFRL/IFSB, 525 Brooks Road, Rome, NY 13441, USA

E-mail: timothy.busch@rl.af.mil

\begin{abstract}
Joint Air Operations (JAO) are traditionally orchestrated using centralised planning and scheduling that assigns air assets to mission packages for the purpose of achieving campaign objectives. However, the current command and control model used by the Air Force lacks 'agility', since it neither anticipate changes in the battlespace nor take advantage of continuous information provided by the sensors in a network-centric environment. In this study, we propose a Multi-Criteria Decision Analysis model that considers four competing objectives (effort, effectiveness, efficiency, and connectivity) to assess vehicle-target allocation for network-centric JAO. The proposed model uses entropy, multi-hop connectivity, and the Analytic Hierarchy Process to derive an overall measures of the performance for alternative vehicle-target allocation scenarios.
\end{abstract}

Keywords: Analytic Hierarchy Process; entropy; Joint Air Operation; JAO; multi-criteria decision analysis; multi-hop connectivity; network-centric; vehicle-target assignment.

Reference to this paper should be made as follows: Tavana, M., Bailey, M.D. and Busch, T.E. (2008) 'A multi-criteria vehicle-target allocation assessment model for network-centric Joint Air Operations', Int. J. Operational Research, Vol. 3, No. 3, pp.235-254.

Biographical notes: Madjid Tavana is a Professor of Management Information Systems and the Lindback Distinguished Chair of Information Systems at the 
La Salle University where he served as the Chairman of the Management Department, Director of the Center for Technology and Management, and the Executive Director of the E-Commerce Institute. He has been a Faculty Fellow in Aeronautics and Space Research at the NASA's Kennedy Space Center, developing Group Decision Support Systems for the Space Shuttle Programme and Johnson Space Center, developing Intelligent Decision Support Systems for Human Exploration of Mars at the Mission Control Center. In 2005, he was awarded the prestigious Space Act Award by NASA. He holds an MBA, a PMIS, and a $\mathrm{PhD}$ in Management Information Systems and received his Post-Doctoral Diploma in Strategic Information Systems from the Wharton School of the University of Pennsylvania. He has published in journals such as Decision Sciences, Interfaces, Information and Management, Computers and Operations Research, among others.

Matthew Bailey is an Assistant Professor of Management at Bucknell University. He received his PhD in Industrial and Operations Engineering from the University of Michigan. His primary research interests are the theory, computation aspects, and applications of stochastic optimisation. In particular, he is interested in modelling adversarial relationships as well as medical decision-making. He is a member of the Institute for Operations Research and the Management Sciences, and the Institute of Industrial Engineers.

Timothy Busch is a Senior Engineer with the Information Systems Research Branch of the Air Force Research Laboratory. He received his $\mathrm{PhD}$ in Electrical Engineering from the Binghamton University in the area of Control Theory. His primary research interest is in the application of model predictive control techniques to dynamic decision problems typical in high tempo operations. He is a research advisor to the National Research Council and an Assistant Professor in the Electrical Engineering Department of the State University of New York Institute of Technology. He is a senior member of the IEEE and also an Associate Editor for the IEEE Transactions on Systems, Man, and Cybernetics Part $C$.

\section{Introduction}

Joint Air Operations (JAO), primarily conducted by the Air Force, involves joint or combined forces in support of a military campaign. JAO are traditionally orchestrated using centralised planning that places the authority for decision-making in one commander who is responsible for developing a coordinated campaign plan for all air operations in the area of responsibility. Air packages (e.g. different vehicles) are assembled and assigned to different geographical locations (e.g. targets) such that their coordinated efforts achieve the JAO objectives. Once the objectives of the mission are defined, Master Air Attack Planners (MAAPs) assign vehicles to targets and develop a plan of execution called the 'air tasking order' detailing how air power will support the overall mission in the presence of uncertainties and a hostile enemy. The use of the term targets in this paper is meant to include all geographical mission assignments and surveillance of points of interest or reconnaissance of named areas.

The air tasking order transmitted to operators at the tactical level lacks 'agility', since it is a static plan produced on a fixed schedule. Changes to the air tasking order are manpower intensive and often require extensive coordination. This severely impacts the ability to take advantage of the continuous information provided by the sensors. The main 
difficulty in developing agile air tasking orders is in understanding the impact of dynamic changes to the plan acerbated by the lack of capabilities for sharing and accessing information. There is a critical need for enhanced real-time information sharing and performance measurement that incorporates anticipative capabilities regarding alternative configuration scenarios and future uncertain events.

The Air Force is exploring various innovative infrastructures to increase the effectiveness of JAO. Network-centric operations have provided JAO with the capability to generate precise effects by accessing new and previously unreachable regions of the information domain. This study exploits this real-time information and proposes a MultiCriteria Decision Analysis (MCDA) model in a network-centric environment to assess different vehicle-target assignment scenarios and derive an effective operational tempo for successful JAO.

The rigid air tasking process does not allow aircrafts to use real-time information to self-organise and more effectively and efficiently engage targets. We propose a dynamic environment where events such as the destruction or discovery of a target or air defence system or loss of a vehicle triggers the assessment system. The system proposed in this study, utilises real-time information via a robust network to enhance information sharing, collaboration, shared situational awareness, and self-synchronisation fundamental to mission effectiveness in network-centric warfare.

The problem of assigning targets to fight units (i.e. aircrafts, battleships, or weapons) has received considerable attention in the operations research community (Manne, 1958; DenBroeder, Ellison and Emerling, 1959; Braford, 1961; Day, 1966; Matlin, 1970; Li, Curry and Boyd, 2004). Several extensive literature surveys are available on the subject (Eckler and Burr, 1972; Murphey, 1999; Voss, 1999). Most of these problems are formulated as optimisation problems where optimum assignment of targets is determined by maximising objective functions such as the expected number of targets destroyed. There are two types of target assignment problems, the static and dynamic problems. In the static problem, all targets are assigned at one time and the objective is to minimise the total expected number of the surviving targets. In the dynamic problem, weapons are assigned to targets in several discrete time intervals and the outcome of previous engagements is used in making future assignments.

Target assignment research to date has focused on developing heuristics or solving special case problems such as identical weapons problems (DenBroeder, Ellison and Emerling, 1959; Katter, 1986) or single weapon problems (Chang, James and Shaw, 1987; Orlin, 1987). Metler and Preston (1990) have studied a series of algorithms for solving the weapon and target assignment problems efficiently, which is critical for realtime applications. Other more complicated algorithms have been developed to solve dynamic target assignment problems (Hosein, Walton and Athans, 1988; Murphey, 1999). While the traditional target assignment models in operations research consider both static and dynamic models, they assume a single role for each vehicle and do not consider a communication objective among the vehicles necessary for successful network-centric operations.

Non-linear programming (Day, 1966), goal programming (Green, Moore and Borsi, 1997), non-linear network flow (Castanon, 1987), neural networks (Wachholder, 1989), and evolutionary genetic algorithms (Grant, 1993; Lee and Lee, 2003, 2005; Lee, Lee and Lee, 2003) are among some of the widely used techniques for solving target assignment problems. The Genetic algorithm models have considered multiple roles for vehicles in a target assignment problem but none have taken into consideration the value of 
communication. Dynamic programming (Flint, 2003) and network optimisation (O'Rourke et al., 2000) are also used to solve target assignment problems. Network optimisation modelling treats the individual vehicles as discrete supplies of single units while tasks are carried out as flows on arcs through the network. Tabu search is used to solve the difficult combinatorial network optimisation problems (O'Rourke et al., 2000; Toth and Vigo, 2002). While these methods are dynamic, they value each vehicle-target assignment independently with a separable objective function.

Beard, McLain and Goodrich (2002) have developed a system for the target assignment and coordinated intercept problem. They solve the target assignment problem by using satisficing and social welfare paradigms to assign unmanned air vehicles to a set of targets. While their model is dynamic and considers multiple roles for each vehicle, it does not take into consideration the value of communication. The model presented in this paper differs from the existing models and has four distinctive characteristics. Our model is dynamic, since the outcome of previous engagements is used in making future assignments, it allows multiple roles for the vehicles, it considers a series of competing objectives simultaneously, and it takes into account the value of communication. All of these factors are considered in the MCDA model. The MCDA model presented here is a solution evaluation tool that can be embedded within common meta-heuristics (see Reeves, 1993) such as genetic algorithms (Holland, 1975; Goldberg, 1989), simulated annealing (Van Laarhoven and Aarts, 1987), and Tabu search (Glover and Laguna, 1996).

We formulate the problem of assigning fighting units (aircraft referred to as 'vehicles' in our study) to targets as the MCDA problem with competing and conflicting objectives (i.e. maximising the number of vehicles assigned to each target, maximising the number of targets assigned to vehicles, minimising the distance between a vehicle and the targets, maximising the distance between a vehicle and the air defence sites, etc.). We develop a weighted-sum overall measure of performance for vehicle-target assignments by integrating different measures reflecting multiple objectives driven by problem situation and not by a particular method. The weighted sum (also called weighted average sum or linear weighted attribute) is a very popular and simple aggregation method (Triantaphyllou, 2000). Each objective is assigned a weighting value representing the relative importance of that objective. The method transforms multiple objectives into an aggregated objective function by multiplying the performance score of each objective by the weighting values and summing up all weighted scores. The weights used in the weighted sum method are selected by the decision-maker(s) prior to determination of the solution. Often experienced decision-makers have difficulty reliably selecting specific values even if they are intimately familiar with the problem domain. The solutions in weighted sum method are strongly dependent upon how the weights were chosen. We use Analytic Hierarchy Process (AHP) introduced by Saaty (1977) to derive the relative importance of each objective. The details of the AHP and the mathematical details of our model are presented in Section 2. We present an application problem in Section 3, followed by the results of the sensitivity analysis in Section 4, and conclusion and future research directions in Section 5. 


\section{Vehicle-target allocation model}

Assuming a vehicle is assigned to one and only one target while multiple vehicles could be assigned to a target, we consider a problem with $T$ targets and $V$ vehicles. Let $h_{v t}$ represent the assignment of the vth vehicle to the th target, where $h_{v t}=1$ if the $v$ th vehicle is assigned to the tth target, otherwise $h_{v t}=0 . H$ is the resulting assignment matrix where the $v$ th row corresponds to the $v$ th vehicle and the th column corresponds to the th target, i.e. $[H]_{v t}=h_{v t}$. The vehicle's role is dependent upon whether the target is confirmed. Although the locations of the targets are known, the current viability of a target must be confirmed before a strike. If the target is confirmed, the vehicle is assigned the 'strike' role and if the target is unconfirmed, the vehicle is assigned the 'sensor' role. There are four competing objectives $\left(F_{r}\right)$ that affect how vehicles are assigned to targets:

- Effort $\left(F_{1}\right)$ : maximise the number of vehicles assigned to each target.

- Effectiveness $\left(F_{2}\right)$ : maximise the number of targets assigned to vehicles.

- Efficiency $\left(F_{3}\right)$ : a combined measure of travel and threats used to minimise the distance between a vehicle and the targets and maximise the distance between a vehicle and the air defence sites.

- Connectivity $\left(F_{4}\right)$ : a measure of multi-hop connectivity used to assess the quality of the current communication network.

Each objective is calculated and normalised to a real number between 0 and 1 . The weighted sum approach considers all objectives as having the same optimisation criterion (minimisation or maximisation). For this purpose, each objective is set to be maximised through inversion where necessary. By means of the linear combination of all the weighted objectives, a combined objective function value $F$ where $0 \leq F \leq 1$ is achieved: $F=\sum_{r=1}^{4} w_{r} F_{r} \cdot w_{r}$ is the relative importance (weight) of each objective $\left(0 \leq w_{r} \leq 1\right)$ where $\sum_{r=1}^{4} w_{r}=1$ and $F_{r}$ is the performance measure of each objective $\left(0 \leq F_{r} \leq 1\right)$. In practice, there is no real need for the $w_{r}$ terms to sum to one. However, this constraint is convenient, since all objective functions are similarly scaled from 0 to 1 .

The overall measure of performance, $F$, developed here is used to evaluate alternative vehicle-target assignment scenarios. A selected scenario is considered robust if the solutions obtained for different possible importance weights associated with the competing objectives remain stable. If the solution model is robust to the values of the importance weights, the level of confidence in the selected scenario is increased significantly. In order to assess the robustness of our model, we perform sensitivity analysis on the importance weights and the performance values of the four competing objectives using the method proposed by Triantaphyllou and Sanchez (1997) for weighted sum MCDA models. We consider $M$ scenarios and $r$ competing objectives. Scenarios will be denoted as $S_{x}$ (for $x=1,2, \ldots, M$ ) and objectives as $F_{r}$ (for $r=1,2,3$ and 4). Furthermore, using $w_{r}$ (for $r=1,2,3$ and 4) as the importance weight of each objective, we assume that $S_{X, r}$ is the measure of performance of scenario $S_{X}$ in terms of objective $F_{r}$ and $F^{x}$ is the weighted overall performance of each scenario. We measure the minimum quantity that the weight for objective $F_{r}$ needs to be changed to reverse the ranking for each pair of scenarios $\left(S_{x}, S_{y}\right)$ under consideration as $\delta_{r, x, y}=F^{y}-F^{x} / s_{y, r}-s_{x, r}$ where $F^{x}$ and $F^{y}$ are the weighted overall performance measures of $S_{x}$ and $S_{y}$. The following condition must be satisfied for the weight to be feasible: $\delta_{r, x, y} \leq w_{r}$. In cases 
where it is possible to reverse the existing ranking $\left(\delta_{r, x, y}>w_{r}\right)$, the modified weight of the first objective is calculated as: $w_{r}^{*}=w_{r}-\delta_{r, x, y}$. The percentage change in the weight can also be calculated as $\% w_{r, x, y}=w_{r}^{*} / w_{r} \times 100$. The threshold value, $\% R_{r, x, y}$, by which the performance measure of scenario $S_{X}$ in terms of objective $F_{r}$, denoted as $S_{X, r}$, needs to be modified so that the ranking of objective $F^{x}$ and $F^{y}$ will be reversed, is given as: $\% R_{r, x, y}=F^{x}-F^{y} / w_{r} \times 100 / s_{x, r}$. Furthermore, the following condition should be satisfied for the threshold value to be feasible: $\% R_{r, x, y} \leq 100$.

To measure effort $\left(F_{1}\right)$, we define $X_{t}$ as the number of vehicles assigned to target $t$, where $X_{t}=\sum_{v=1}^{V} h_{v t}$. In addition, we define $p_{t}$ as the proportion of total vehicles assigned to target $t$, i.e. $p_{t}=X_{t} / V$. We further assume $w_{t}^{\prime}$ is the weight associated with each confirmed or unconfirmed target where $\sum_{t=1}^{T} w_{t}^{\prime}=1$. The importance weight associated with each target can be elicited by different weighting procedures. The simplest way is weighting each target directly by point allocation. Other weighting methods include SMART and SMARTER (Edwards and Barron, 1994; Barron and Barnett, 1996), SWING (Von Winterfeldt and Edwards, 1986), and AHP (Saaty, 1977, 1983, 1994). Using SMART, 10 points are given to the least important target. Then, more points are given to the other targets, depending upon their relative importance. In SMARTER, the weights are elicited with the centroid method of Solymosi and Dombi (1986). The SWING method is similar, but the procedure starts from the most important target, keeping it as the reference.

In this model, we assume the priority of the targets are known and given. This is not unreasonable, since the current process produces a Joint Integrated Prioritised Target List. However, we could have used AHP to elicit the importance weights of the targets as we do later in this study with the importance weight of the objectives in our MCDA model. We find $\bar{p}_{t}$, the weighted proportion of total vehicles assigned to target $t$ as $\bar{p}_{t}=w_{t}^{\prime} p_{t}$. Next, we find the normalised weighted proportion of total vehicles assigned to target $t$, $\hat{p}_{t}$, where $\hat{p}_{t}=\bar{p}_{t} / \sum_{j=1}^{T} \bar{p}_{j}$. Using the vector $\hat{p}=\left(\hat{p}_{1}, \hat{p}_{2}, \ldots, \hat{p}_{T}\right)$, a Shannon entropy measure (Shannon, 1948), $E(\hat{p})=-1 / \ln (r) \sum_{t=1}^{T} \hat{p}_{t} \ln \hat{p}_{t}$ is used to evaluate the amount of dissonance among the weighted proportion of vehicles assigned to different targets. This entropy measure takes a value between 0 and 1 . Its value is zero if all vehicles are assigned to a target with maximum weight and its value is one if the weighted proportion assigned to each target is identical. Our efficiency measure, $F_{1}$, is then defined as $F_{1}=1-E(\hat{p})$ where $0 \leq F_{1} \leq 1$. The higher $F_{1}$, the more vehicles are assigned to higher-valued targets.

To measure effectiveness $\left(F_{2}\right)$, we find the proportion of the total value of targets that are assigned at least one vehicle. We define $b_{t}=1$ if $h_{v t}=1$ for some $v$ and $b_{t}=0$ otherwise, then $F_{2}=\sum_{t=1}^{T} w_{t}^{\prime} b_{t}$ and $0 \leq F_{2} \leq 1$.

To measure efficiency $\left(F_{3}\right)$, we define $d_{v t}$ as the distance of the $v$ th vehicle to the th target. Since we are maximising this measure, we define $\bar{d}_{v t}, \bar{d}_{v t}=1 / d_{v t}$ and $\hat{d}_{v t}$, the normalised measure of performance of distance of the $v$ th vehicle to the th target, as $\hat{d}_{v t}=\bar{d}_{v t} / \sum_{t=1}^{T} \sum_{v=1}^{V} \bar{d}_{v t}$. Let us further consider $A$ air defence sites and define $d_{v a}$ as the 
distance of the $v$ th vehicle to the $a$ th air defence site. The average distance of the $v$ th vehicle to the $A$ air defence sites, $\bar{d}_{v}$, is $\bar{d}_{v}=\sum_{a=1}^{A} d_{v a} / A$. Further defining $d_{t a}$ as the distance of the th target to the $a$ th air defence site, we find the average distance of target $t$ to the $A$ air defence sites, $\bar{d}_{t}$, as $\bar{d}_{t}=\sum_{a=1}^{A} d_{t a} / A$. The measure of performance of assigning $v$ th vehicle to the th target in terms of average distance from the air defence site, $\overline{\bar{d}}_{v t}$, is $\overline{\bar{d}}_{v t}=\bar{d}_{v}+\bar{d}_{t} / 2$. We find the normalised measure of this performance as $\hat{\hat{d}}=\overline{\bar{d}}_{v t} / \sum_{t=1}^{T} \sum_{v=1}^{V} \overline{\bar{d}}_{v t}$. Next, we average $\hat{d}_{v t}$ and $\hat{\hat{d}}$ to find $d_{v t}^{*}$, the combined measure of travel and threat for assigning the $v$ th vehicle to the th target $\left(d_{v t}^{*}=\hat{d}_{v t}+\hat{\hat{d}}_{v t} / 2\right)$. Using these values, we find the normalised measure of travel and threat for assigning the vth vehicle to the tth-target, $d_{v t}^{* *}=d_{v t}^{*}-\underset{v, t}{\operatorname{Min}} d_{v t}^{*} / \underset{v, t}{\operatorname{Max}} d_{v t}^{*}-\underset{v, t}{\operatorname{Min}} d_{v t}^{*}$. The overall measure of travel and threat for a given assignment, $F_{3}$, is $F_{3}=\sum_{v, t} h_{v t} d_{v t}^{* *} / V$ where $0 \leq F_{3} 1$.

To measure connectivity $\left(F_{4}\right)$, we assess the quality of the current communication network using a measure of multi-hop connectivity (Miller, 2001). Multi-hop connectivity provides a measure of the number of vehicles (or communication nodes) through which information must be relayed for vehicles to communicate. For $V$ vehicles, we create the $V \times V$ multi-hop connectivity matrix $C$ where the $(i, j)$ th entry in the matrix, $c_{i j}$ corresponds to the minimum number of hops for vehicle $i$ to relay information to vehicle $j$ and a 0 indicates that no connectivity exists between $i$ and $j$. In this instance, we replace 0 with $V+1$ to penalise the disconnectivity. As detailed by Miller (2001), we derive this matrix from an initial adjacency matrix $A$, where a 1 in the $(i, j)$ th entry of this matrix indicates that vehicle $i$ is directly linked to vehicle $j$ and a 0 indicates that no direct connection exists. Once the multi-hop connectivity matrix $C$ has been derived we compute the value $\beta$ where $\beta=\sum_{i=1}^{V} \sum_{j=1}^{1} c_{i j} / V(V-1)$ and $0 \leq \beta \leq V+1$. For fullyconnected networks, is $\beta$ the average hop distance; and for disconnected networks, $\beta$ is the penalised hop distance (penalised for disconnectivity). $F_{4}=1-\beta / V+1$ provides the measure of the connectivity of the current network $\left(0 \leq F_{4} \leq 1\right)$.

The relative importance (i.e. weighting) of the four objectives is captured through a series of pairwise comparisons using the AHP and Expert Choice software. AHP was introduced by Saaty (1977) to assist decision-makers in the evaluation of complex judgmental problems. Saaty argues that a decision-maker naturally finds it easier to compare two things than to compare all the items in a list. AHP also evaluates the consistency of the decision-makers and allows for the revision of their responses. Because of the intuitive nature of the process and its power in resolving the complexity in a judgmental problem, AHP has been applied to many diverse decisions. A comprehensive list of the major applications of AHP, along with a description of the method and its axioms, can be found in Saaty (1977, 1983, 1994), Zahedi (1986) and Weiss and Rao (1987). AHP has proven to be a very popular technique for determining weights in multi-criteria problems and has gained wide acceptance among academics and practitioners (Golden, Wasil and Harker, 1989; Shim, 1989; Forman and Gass, 2001; Golden and Wasil, 2003; Gass, 2005). 
The MAAPs are asked to provide their subjective assessment of each pairwise comparison. Saaty's AHP uses these pairwise comparisons to derive a weight for each objective. This requires each MAAP to make six pairwise comparisons. Let us assume that $c_{1}, C_{2}, C_{3}$, and $c_{4}$ are the four objectives that contribute to the overall quality of a particular vehicle-target assignment. The MAAP's next task is to assess the relative importance of these objectives by comparing each possible pair $\left(c_{j}, c_{k}\right)$ and indicating which objective is more important and by how much. These judgments are represented by a $4 \times 4$ matrix, $\Gamma=\left(\gamma_{j k}\right)$ where $j, k=1, \ldots, 4$. If $c_{j}$ is judged to be of equal importance as $c_{k}$, then $\gamma_{j k}=1$; if $c_{j}$ is judged to be more important than $c_{k}$, then $\gamma_{j k}>1$; and if $c_{j}$ is judged to be less important than $c_{k}$, then $\gamma_{j k}<1 . \gamma_{j k}=1 / \gamma_{j k}$ where $\gamma_{j k} \neq 0$. Thus, matrix $\Gamma$ is a reciprocal matrix so that the entry $\gamma_{j k}$ is the inverse of the entry $\gamma_{k j} \cdot \gamma_{j k}$ reflects the relative importance of $c_{j}$ compared with objective $c_{k}$. For example, $\gamma_{12}=1.25$ indicates that $c_{1}$ was 1.25 times as important as $c_{2}$.

The vector $W$ representing the relative weights of each of the four objectives is found by computing the normalised eigenvector corresponding to the maximum eigenvalue of matrix $\Gamma$. An eigenvalue of $\Gamma$ is defined as $\lambda$ which satisfies the matrix equation $\Gamma w=\lambda w$, where $\lambda$ is a constant, called the eigenvalue, associated with the given eigenvector $w$. Saaty has shown that the best estimate of $w$ is the one associated with the maximum eigenvalue $\left(\lambda_{\max }\right)$ of the matrix $\Gamma$. Because the sum of the weights should be equal to 1.00 , the normalised eigenvector is used. Saaty's algorithm for obtaining this $w$ is incorporated in the software Expert Choice (2004) utilised in this study.

One of the advantages of AHP is that it assesses the consistency of the MAAP's pairwise comparisons. Saaty suggests a measure of consistency for the pairwise comparisons. When the judgments are perfectly consistent, the maximum eigenvalue $\left(\lambda_{\max }\right)$ should equal to the number of objectives that are compared (four). Typically, the responses are not perfectly consistent, and $\lambda_{\max }$ is greater than four. The larger the $\lambda_{\max }$, the greater is the degree of inconsistency. Saaty defines a consistency index as $\left(\lambda_{\max }-4\right) /(4-1)$ and provides a random index table for matrices of order 3 to 10 . This random index is based on a simulation of a large number of randomly generated weights.

The responses of the MAAPs are processed with Expert Choice and those with inconsistency ratios greater than 0.10 are asked to reconsider their judgments as suggested by Saaty $(1977,1983,1994)$. The importance weights are averaged after the necessary adjustments are made to inconsistent responses. Each MAAP is presented with his or her individual score along with the group mean weights. MAAPs are given the opportunity to revisit their judgments and make revisions to their pairwise comparison scores based on this feedback. As a result, a set of weights representing the relative importance of the four objectives is found.

There has been some criticism of AHP in the operations research community. Harker and Vargas (1987) show that AHP does have an axiomatic foundation, the cardinal measurement of preferences is fully represented by the eigenvector method, and the principles of hierarchical composition and rank reversal are valid. On the other hand, Dyer (1990a) has questioned the theoretical basis underlying AHP and argues that it can lead to preference reversals based on the alternative set being analysed. In response, Saaty (1990) explains how rank reversal is a positive feature when new reference points are introduced. The Geometric aggregation rule can be used to avoid rank reversal which has had varying degrees of importance to different researchers (Dyer, 1990a,b; Harker and Vargas, 1990; Saaty, 1990). 


\section{Application problem}

We consider a tactical military operations problem with five vehicles $(A, B, C, D$, and $E$ ), five targets $\left(A^{\prime}, B^{\prime}, C^{\prime}, D^{\prime}\right.$, and $\left.E^{\prime}\right)$, and five air defence sites $\left(A^{\prime \prime}, B^{\prime \prime}, C^{\prime \prime}, D^{\prime \prime}\right.$, and $\left.E^{\prime \prime}\right)$. The distance matrix for the targets and air defence sites are presented in Table 1.

Table 1 The distance matrix for the targets and air defence sites (miles)

\begin{tabular}{|c|c|c|c|c|c|c|}
\hline & & \multicolumn{5}{|c|}{ Air defence site } \\
\hline & & $A^{\prime \prime}$ & $B^{\prime \prime}$ & $C^{\prime \prime}$ & $D^{\prime \prime}$ & $E^{\prime \prime}$ \\
\hline \multirow{5}{*}{ 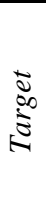 } & $A^{\prime}$ & 15 & 43 & 86 & 145 & 79 \\
\hline & $B^{\prime}$ & 33 & 30 & 51 & 112 & 60 \\
\hline & $C^{\prime}$ & 147 & 120 & 73 & 16 & 95 \\
\hline & $D^{\prime}$ & 130 & 100 & 71 & 25 & 68 \\
\hline & $E^{\prime}$ & 51 & 39 & 86 & 119 & 41 \\
\hline
\end{tabular}

The MAAPs are provided with $0.25,0.2,0.15,0.15$, and 0.1 as the importance weight of targets $A^{\prime}, B^{\prime}, C^{\prime}, D^{\prime}$, and $E^{\prime}$. As discussed earlier, AHP and Expert Choice could have been used to determine the importance weights of the targets. Initially, at time period zero $(t=0)$, all targets were confirmed and all vehicles were assigned the 'strike' role. Vehicles $A$ and $B$ were assigned to target $A^{\prime}$ while vehicles $C, D$, and $E$ were assigned to targets $B^{\prime}, C^{\prime}$, and $D^{\prime}$. No vehicle was assigned to target $E^{\prime}$. The MAAPs also used AHP and Expert choice as a group to determine the importance weight of the four objectives: effort $\left(F_{1}\right)$, effectiveness $\left(F_{2}\right)$, efficiency $\left(F_{3}\right)$, and connectivity $\left(F_{4}\right)$. Alternatively, each MAAP could have used Expert Choice individually to derive his or her importance weights. In that case, the geometric aggregation rule would have been used to combine individual weights into a group weight. Table 2 shows the pairwise comparison matrix along with the importance weights for the four factors derived through brainstorming by the MAAPs. These judgments were considered consistent because the inconsistency ratio associated with the pairwise comparison matrix was zero.

Table 2 The pairwise comparison matrix and the relative weights

\begin{tabular}{lcccc}
\hline & Effort $\left(F_{1}\right)$ & Effectiveness $\left(F_{2}\right)$ & Efficiency $\left(F_{3}\right)$ & Connectivity $\left(F_{4}\right)$ \\
\hline Effort $\left(F_{1}\right)$ & 1.000 & 0.500 & 0.250 & 0.333 \\
Effectiveness $\left(F_{2}\right)$ & 2.000 & 1.000 & 0.500 & 0.625 \\
Efficiency $\left(F_{3}\right)$ & 4.000 & 2.000 & 1.000 & 1.400 \\
Connectivity $\left(F_{4}\right)$ & 3.000 & 1.600 & 0.715 & 1.000 \\
Overall weight & 0.100 & 0.196 & 0.404 & 0.300 \\
\hline
\end{tabular}

Note: Inconsistency ratio $=0$.

At $t+5$ (5 min after takeoff), the initial assignment of vehicles to targets remained unchanged. The distance matrix for the vehicles and targets and the distance matrix for the vehicles and air defence sites are presented in Tables 3 and 4 .

Using the methods described in Section 2 for effort, effectiveness, efficiency, and connectivity, the performance scores of $F_{1}, F_{2}, F_{3}$, and $F_{4}$ were calculated as 0.769 , $0.750,0.421$, and 0.767 . The low score of 0.421 for $F_{3}$ (efficiency) was primarily due to the long distances between the vehicles and targets (see Table 3 and Figure 1 ). Considering the importance weight of the four objectives and their performance scores, we found the overall weighted performance score of this state as 0.624 . The importance 
weights and the performance scores along with a picture of the $t+5$ state are given in Figure 1.

Table 3 The distance matrix for the vehicles and targets at $t+5$ (miles)

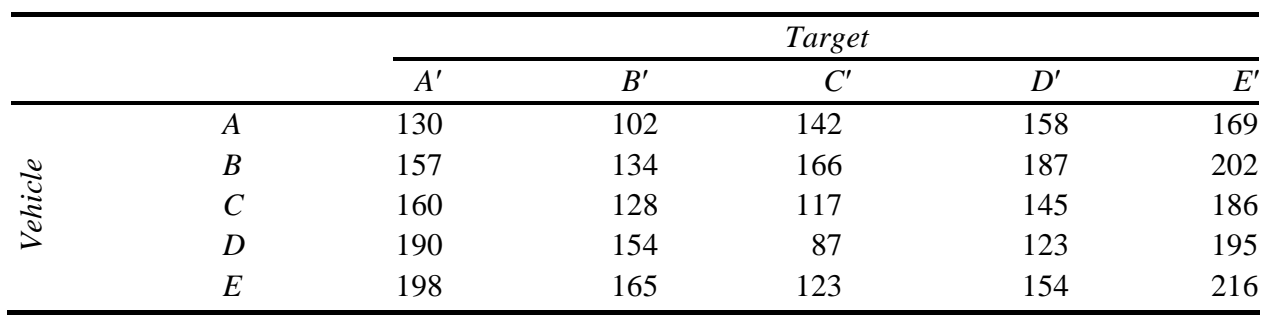

Table 4 The distance matrix for the vehicles and air defence sites at $t+5$ (miles)

\begin{tabular}{|c|c|c|c|c|c|c|}
\hline & & \multicolumn{5}{|c|}{ Air defence sites } \\
\hline & & $A^{\prime \prime}$ & $B^{\prime \prime}$ & $C^{\prime \prime}$ & $D^{\prime \prime}$ & $E^{\prime \prime}$ \\
\hline \multirow{5}{*}{$\frac{\frac{v}{v}}{\frac{d}{d}}$} & $A$ & 132 & 128 & 96 & 140 & 145 \\
\hline & $B$ & 164 & 160 & 128 & 167 & 176 \\
\hline & C & 162 & 150 & 103 & 122 & 154 \\
\hline & $D$ & 187 & 166 & 112 & 98 & 157 \\
\hline & $E$ & 200 & 183 & 132 & 135 & 182 \\
\hline
\end{tabular}

Figure 1 The $t+5$ state (For colour figures, please see online version)

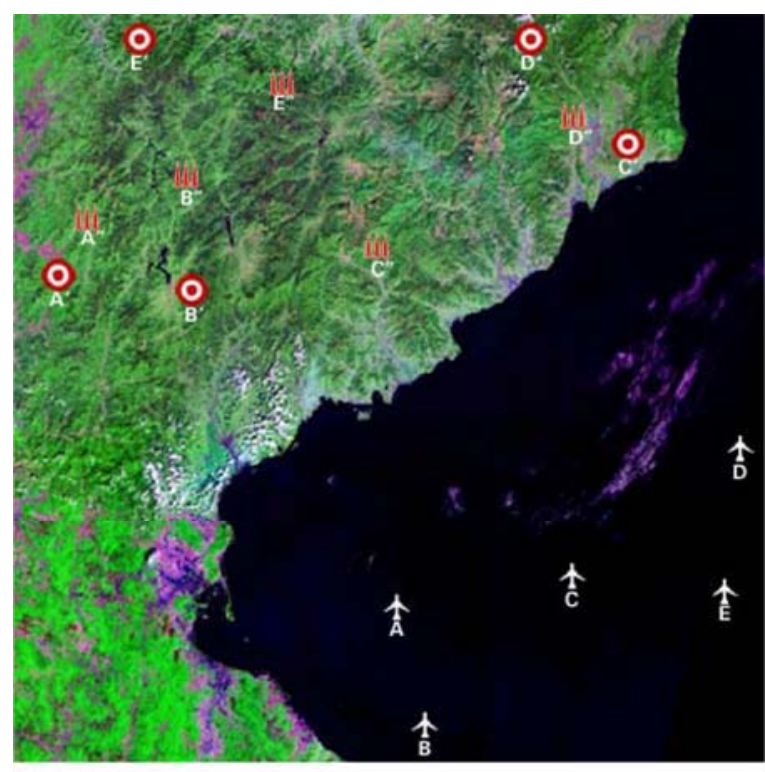

\begin{tabular}{|c|c|c|c|c|}
\hline $\begin{array}{c}\text { Performance } \\
\text { Measures }\end{array}$ & $\boldsymbol{F 1}$ & $\boldsymbol{F 2}$ & $\boldsymbol{F 3}$ & $\boldsymbol{F 4}$ \\
\hline Importance Weight & .100 & .196 & .404 & .300 \\
\hline Performance Score & .769 & .750 & .421 & .767 \\
\hline
\end{tabular}


At $t+15$, the initial assignment of vehicles to targets remained unchanged. Given the new vehicle-target distance matrix and vehicle-air defence site distance matrix, we measured the performance score of $F_{1}, F_{2}, F_{3}$, and $F_{4}$ and the overall weighted performance score of the state. As it is shown in Figure 2, $F_{1}$ and $F_{2}$ remained unchanged (0.769 and 0.750, respectively). However, $F_{3}$ was decreased to 0.332 (a reduction of 21\%) because while the vehicles were closer to the targets, they were also much closer to the air defence sites. In addition, $F_{4}$ was decreased to 0.667 (a reduction of 13\%) because of the increase in the number of vehicles (or communication nodes) through which information must be relayed for vehicles to communicate.

Figure 2 The $t+15$ state

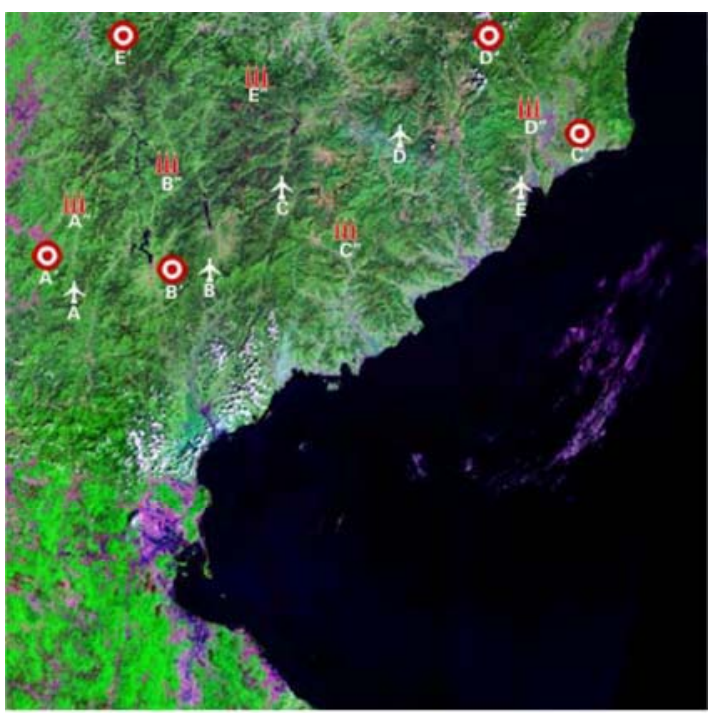

\begin{tabular}{|c|c|c|c|c|c|c|}
\hline \multirow{2}{*}{\multicolumn{2}{|c|}{$\begin{array}{c}\text { Vehicle-Target } \\
\text { Distance Matrix } \\
\text { (miles) }\end{array}$}} & \multicolumn{5}{|c|}{ Target } \\
\hline & & $A^{\prime}$ & $B^{\prime}$ & $C^{\prime}$ & $D^{\prime}$ & $E^{\prime}$ \\
\hline \multirow{5}{*}{$\frac{\frac{2}{\varrho}}{\frac{\pi}{2}}$} & $A$ & 12 & 28 & 153 & 142 & 75 \\
\hline & $B$ & 47 & 11 & 115 & 105 & 72 \\
\hline & C & 70 & 40 & 87 & 84 & 63 \\
\hline & $D$ & 108 & 76 & 52 & 38 & 85 \\
\hline & $E$ & 139 & 104 & 23 & 45 & 123 \\
\hline
\end{tabular}

\begin{tabular}{|c|c|c|c|c|c|c|}
\hline \multirow{2}{*}{\multicolumn{2}{|c|}{$\begin{array}{c}\text { Vehicle-Air } \\
\text { Defense Site } \\
\text { Distance Matrix } \\
\text { (miles) }\end{array}$}} & \multicolumn{5}{|c|}{ Air Defense Sites } \\
\hline & & $A^{\prime \prime}$ & $B^{\prime \prime}$ & $C^{\prime \prime}$ & $D^{\prime \prime}$ & $E^{\prime \prime}$ \\
\hline \multirow{5}{*}{ 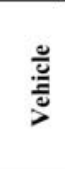 } & $A$ & 25 & 45 & 80 & 142 & 82 \\
\hline & $B$ & 43 & 32 & 40 & 104 & 56 \\
\hline & C & 61 & 34 & 23 & 75 & 33 \\
\hline & $D$ & 97 & 68 & 33 & 38 & 45 \\
\hline & $E$ & 130 & 103 & 52 & 23 & 83 \\
\hline
\end{tabular}

\begin{tabular}{|c|c|c|c|c|}
\hline Performance Measures & $\boldsymbol{F 1}$ & $\boldsymbol{F 2}$ & $\boldsymbol{F 3}$ & $\boldsymbol{F 4}$ \\
\hline Importance Weight & .100 & .196 & .404 & .300 \\
\hline Performance Score & .769 & .750 & .332 & .667 \\
\hline
\end{tabular}


At $t+15$, the MAAPs considered changing the $A-A^{\prime}, B-A^{\prime}, C-B^{\prime}, D-C^{\prime}$, and $E-D^{\prime}$ assignment of vehicles to targets. Three scenarios were considered: $t+15 \mathrm{~A}, t+15 \mathrm{~B}$, and $t+15 \mathrm{C}$. The performance measures for the three scenarios are presented in Table 5 . As discussed earlier in Section 1, meta-heuristics such as genetic algorithms, simulated annealing, and Tabu search could be used to find additional or the optimal solution using the evaluation model presented here. Scenario $t+15 \mathrm{~A}$ was considered by switching $A-B^{\prime}, B-B^{\prime}$, and $C-A^{\prime}$. As a result of this new configuration, $F_{3}$ was improved by $7 \%$ to 0.354 and the overall weighted performance score was improved by $2 \%$ to 0.567 . Next, Scenario $t+15 \mathrm{~B}$ was considered by switching $D-D^{\prime}$ and $E-C^{\prime}$. This new configuration resulted in a $22 \%$ improvement in $F_{3}$ to 0.405 and a $5 \%$ improvement in the overall weighted performance score of the state to 0.587. Finally, the MAAPs considered combining both scenarios $t+15 \mathrm{~A}$ and $t+15 \mathrm{~B}$ into one scenario, $t+15 \mathrm{C}$ by switching $A-B^{\prime}$, $B-B^{\prime}, C-A^{\prime}, D-D^{\prime}$ and $E-C^{\prime}$. The combined configuration resulted in a $28 \%$ improvement in $F_{3}$ to 0.426 and a $7 \%$ improvement in the overall weighted performance score of the state to 0.596 . Configuration $t+15 \mathrm{C}$ was selected by the MAAPs and the vehicles received their new assignments.

Table 5 The $t+15$ alternative assignments

Alternative assignment $t+15 \mathrm{~A}$

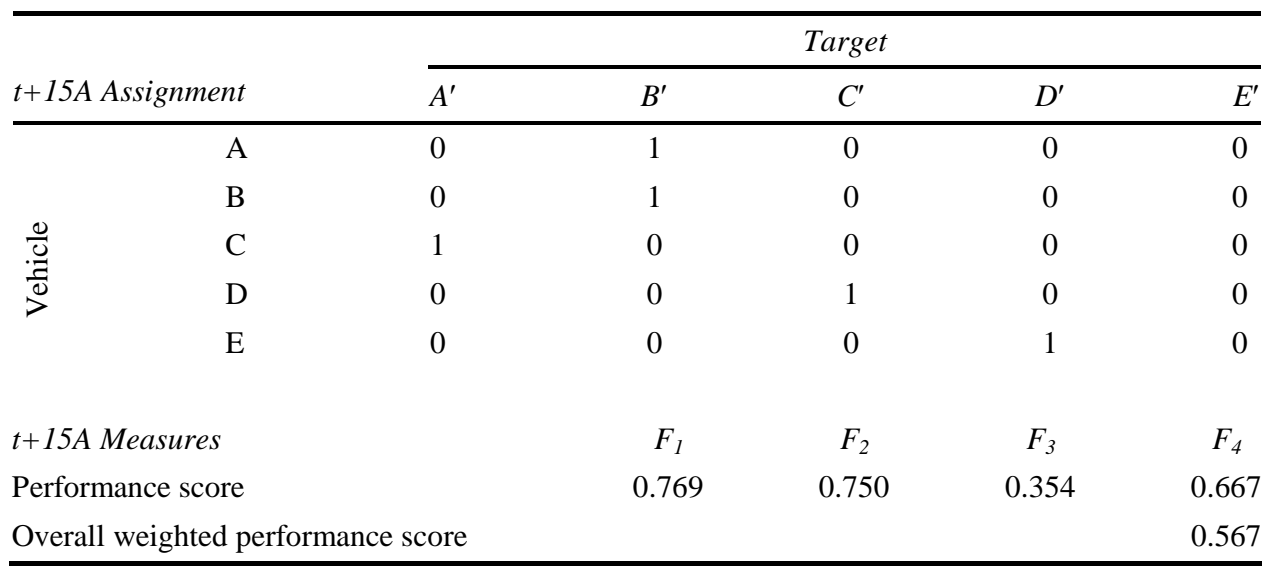

Alternative assignment $t+15 B$

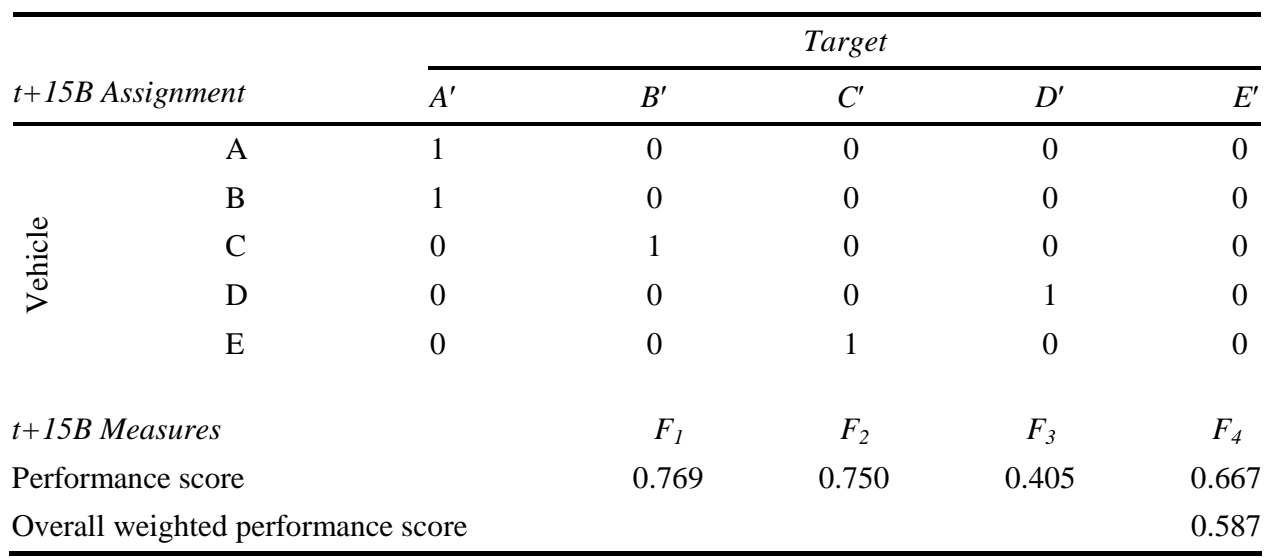


Table 5 The $t+15$ alternative assignments (continued)

Alternative assignment $t+15 C$

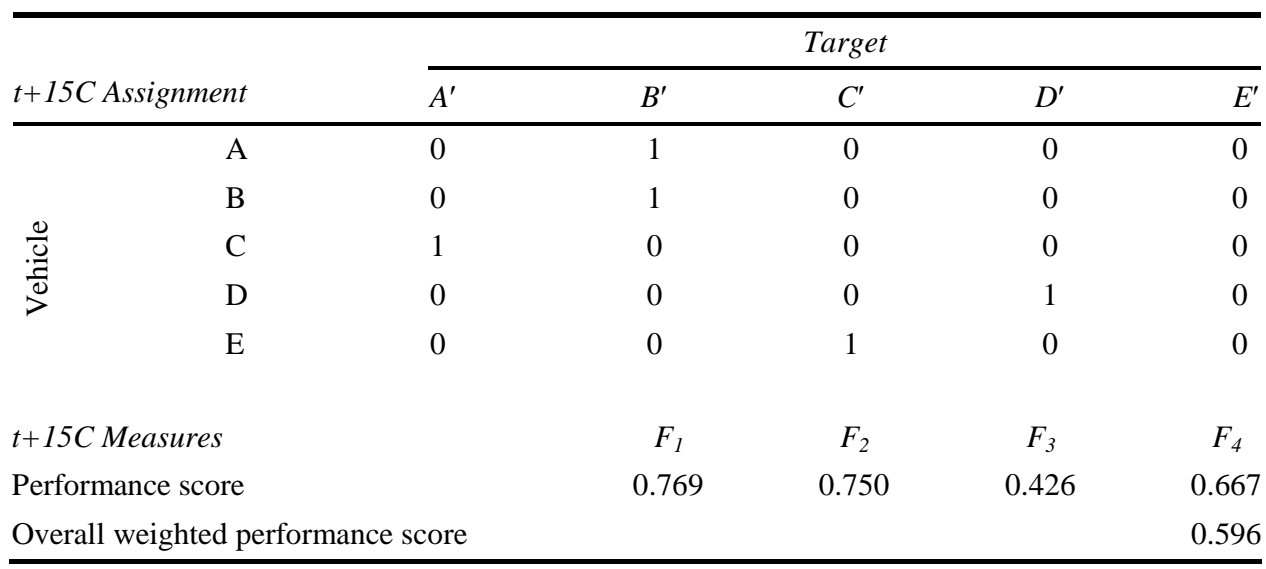

At $t+25$, the vehicles engaged the targets and targets $A^{\prime}$ and $B^{\prime}$ were attacked but not yet verified as destroyed. The assignment of vehicles to target remained unchanged as $A-B^{\prime}$, $B-B^{\prime}, C-A^{\prime}, D-D^{\prime}$ and $E-C^{\prime}$. The distance matrix for the vehicles and targets and the distance matrix for the vehicles and air defence sites along with a picture of the $t+25$ state are given in Figure 3. As shown, the overall weighted performance score of this state was 0.463 .

Given the new information concerning the attack on targets $A^{\prime}$ and $B^{\prime}$, the MAAPs revisited the priority scores of the targets. Targets $A^{\prime}$ and $B^{\prime}$ were downgraded and received a reduced priority score with their remaining scores reallocated among targets $C^{\prime}, D^{\prime}$, and $E^{\prime}$ through normalisation. The new priority score vector for targets $A^{\prime}, B^{\prime}, C^{\prime}$, $D^{\prime}$, and $E^{\prime}$ was $0.05,0.05,0.3375,0.3375$, and 0.225 . In light of the new information, the MAAPs also revisited the assignment of vehicles to targets by considering two scenarios: $t+25 \mathrm{~A}$ and $t+25 \mathrm{~B}$.

In scenario $t+25 \mathrm{~A}$, vehicle $B$ was assigned to target $E^{\prime}$. As a result, the performance score of $F_{1}$ was reduced by $11 \%$ to 0.642 because none of the targets have more that one vehicle assigned to them and the performance score of $F_{2}$ was increased by $29 \%$ to 1.000 because all targets were assigned to at least one vehicle. $F_{3}$ was also improved by $21 \%$ to 0.117 because of the revised priority score of the targets. The overall weighted performance score of the $t+25 \mathrm{~A}$ scenario was improved by $10 \%$ to 0.507 compared to the 0.463 overall weighted performance score at $t+25$. The MAAPs also considered scenario $t+25 B$ by assigning $A-C^{\prime}, B-D^{\prime}$, and $E-E^{\prime}$. In this configuration, $F_{1}$ was slightly decreased by $3 \%$ to 0.696 while $F_{2}$ and $F_{3}$ were increased significantly by 23 and $400 \%$ to 0.950 and 0.492 , respectively. The overall weighted performance score of $t+25 \mathrm{~B}$ was also improved significantly by $42 \%$ to 0.655 . The MAAPs chose this alternative. In this configuration, vehicle $C$ was assigned to target $A^{\prime}$ which was previously attacked but not verified. This assignment implies a new role allocation for vehicle $C$ from 'strike' to 'sensor'. All vehicles were informed about their new target and role assignments. The numerical performance scores for these two scenarios are presented in Table 6. 
Figure 3 The $t+25$ state

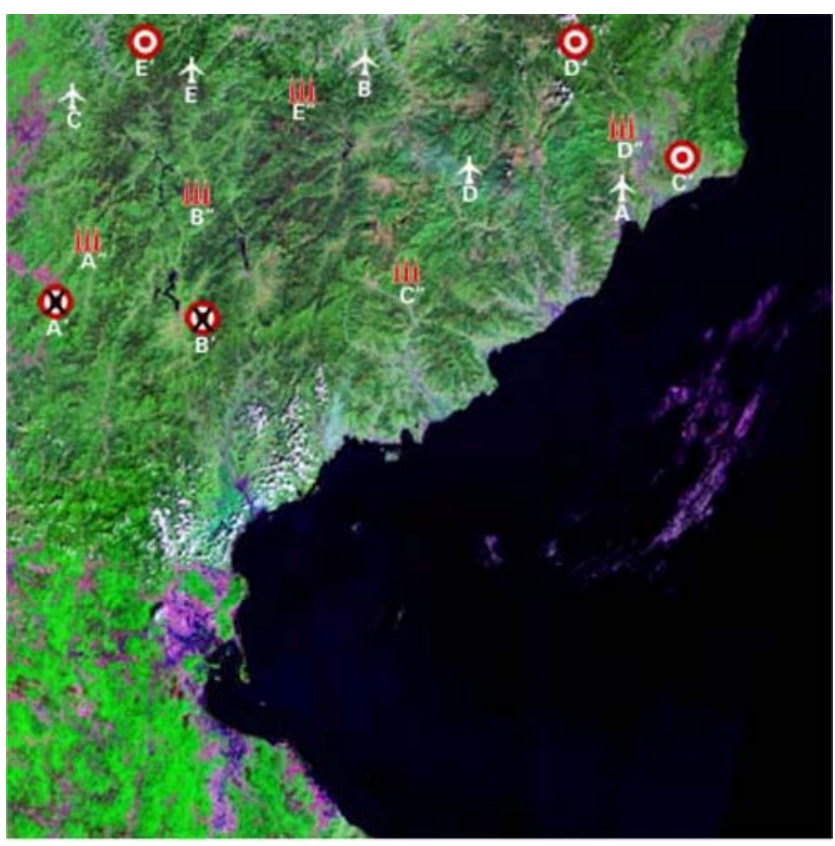

\begin{tabular}{|c|c|c|c|c|c|c|}
\hline \multirow{2}{*}{\multicolumn{2}{|c|}{$\begin{array}{c}\text { Vehicle-Target } \\
\text { Distance Matrix } \\
\text { (miles) }\end{array}$}} & \multicolumn{5}{|c|}{ Target } \\
\hline & & $A^{\prime}$ & $B^{\prime}$ & $C^{\prime}$ & $D^{\prime}$ & $E^{\prime}$ \\
\hline \multirow{5}{*}{ 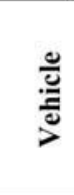 } & $A$ & 142 & 108 & 16 & 37 & 123 \\
\hline & $B$ & 97 & 75 & 82 & 52 & 54 \\
\hline & $C$ & 52 & 64 & 151 & 124 & 22 \\
\hline & $D$ & 107 & 75 & 53 & 40 & 85 \\
\hline & $E$ & 68 & 63 & 123 & 94 & 13 \\
\hline
\end{tabular}

\begin{tabular}{|c|c|c|c|c|c|c|}
\hline \multirow{2}{*}{\multicolumn{2}{|c|}{$\begin{array}{c}\text { Vehicle-Air } \\
\text { Defense Site } \\
\text { Distance Matrix } \\
\text { (miles) }\end{array}$}} & \multicolumn{5}{|c|}{ Air Defense Sites } \\
\hline & & \multirow{2}{*}{$\begin{array}{c}A^{\prime \prime} \\
133\end{array}$} & \multirow{2}{*}{$\begin{array}{l}B^{\prime \prime} \\
105\end{array}$} & \multirow{2}{*}{$\begin{array}{l}C^{\prime \prime} \\
57\end{array}$} & \multirow{2}{*}{$\begin{array}{l}D^{\prime \prime} \\
13 \\
\end{array}$} & \multirow{2}{*}{$\begin{array}{l}E^{\prime \prime} \\
82 \\
\end{array}$} \\
\hline \multirow{5}{*}{ 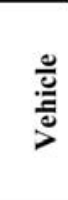 } & $A$ & & & & & \\
\hline & $B$ & 82 & 53 & 54 & 65 & 17 \\
\hline & $C$ & 35 & 37 & 93 & 135 & 56 \\
\hline & $D$ & 95 & 67 & 30 & 37 & 45 \\
\hline & $E$ & 50 & 31 & 73 & 107 & 27 \\
\hline
\end{tabular}

\begin{tabular}{|c|c|c|c|c|}
\hline Performance Measures & $\boldsymbol{F 1}$ & $\boldsymbol{F 2}$ & $\boldsymbol{F 3}$ & $\boldsymbol{F 4}$ \\
\hline Importance Weight & .100 & .196 & .404 & .300 \\
\hline Performance Score & 0.719 & 0.775 & 0.097 & 0.667 \\
\hline
\end{tabular}


Table 6 The $t+25$ alternative assignments

Alternative assignment $t+25 A$

\begin{tabular}{|c|c|c|c|c|c|c|}
\hline \multirow{2}{*}{\multicolumn{2}{|c|}{$t+25$ A Assignment }} & \multicolumn{5}{|c|}{ Target } \\
\hline & & $A^{\prime}$ & $B^{\prime}$ & $C^{\prime}$ & $D^{\prime}$ & $E^{\prime}$ \\
\hline \multirow{5}{*}{$\begin{array}{l}\frac{0}{v} \\
\frac{0}{d}\end{array}$} & A & 0 & 1 & 0 & 0 & 0 \\
\hline & $\mathrm{B}$ & 0 & 0 & 0 & 0 & 1 \\
\hline & $\mathrm{C}$ & 1 & 0 & 0 & 0 & 0 \\
\hline & $\mathrm{D}$ & 0 & 0 & 0 & 1 & 0 \\
\hline & $\mathrm{E}$ & 0 & 0 & 1 & 0 & 0 \\
\hline \multicolumn{3}{|c|}{$t+25 A$ Measures } & $F_{1}$ & $F_{2}$ & $F_{3}$ & $F_{4}$ \\
\hline \multicolumn{3}{|c|}{ Performance score } & 0.642 & 1.000 & 0.117 & 0.667 \\
\hline \multicolumn{3}{|c|}{ Overall weighted performance score } & & & & 0.507 \\
\hline
\end{tabular}

Alternative assignment $t+25 B$

\begin{tabular}{|c|c|c|c|c|c|c|}
\hline \multirow{2}{*}{\multicolumn{2}{|c|}{ t+25B Assignment }} & \multicolumn{5}{|c|}{ Target } \\
\hline & & $A^{\prime}$ & $B^{\prime}$ & $C^{\prime}$ & $D^{\prime}$ & $E^{\prime}$ \\
\hline \multirow{5}{*}{$\begin{array}{l}\frac{\ddot{J}}{\tilde{J}} \\
\stackrel{0}{\nu}\end{array}$} & $\mathrm{A}$ & 0 & 0 & 1 & 0 & $\overline{0}$ \\
\hline & B & 0 & 0 & 0 & 1 & 0 \\
\hline & $\mathrm{C}$ & 1 & 0 & 0 & 0 & 0 \\
\hline & $\mathrm{D}$ & 0 & 0 & 0 & 1 & 0 \\
\hline & $\mathrm{E}$ & 0 & 0 & 0 & 0 & 1 \\
\hline \multicolumn{3}{|c|}{$t+25 B$ Measures } & $F_{1}$ & $F_{2}$ & $F_{3}$ & $F_{4}$ \\
\hline \multicolumn{3}{|c|}{ Performance score } & 0.696 & 0.950 & 0.492 & 0.667 \\
\hline \multicolumn{3}{|c|}{ Overall weighted performance score } & & & & 0.655 \\
\hline
\end{tabular}

\section{Sensitivity analysis}

In order to assess the robustness of our results, we perform sensitivity analysis on the importance weights and the performance values of the four objectives using the method of Triantaphyllou and Sanchez (1997) described earlier.

At $t+15$, we considered three scenarios, $S_{1}(t+15 \mathrm{~A}), S_{2}(t+15 \mathrm{~B})$, and $S_{3}(t+15 \mathrm{C})$. The weights of the four evaluation factors $\left(F_{1}, F_{2}, F_{3}\right.$, and $\left.F_{4}\right)$ were $0.100,0.196,0.404$, and 0.300 , respectively. Each scenario had a performance score on each factor. For example, $S_{1}$ had a score of $0.769,0.750,0.332$, and 0.667 on $F_{1}, F_{2}, F_{3}$, and $F_{4}$, respectively. The overall weighted performance score was calculated as the weighted sum of the performance scores. The overall weighted performance score of $S_{1}, S_{2}$, and $S_{3}$ were 0.567, 0.587, and 0.596, respectively (see Table 7.1). $S_{3}$ was preferred to $S_{2}$, and, $S_{2}$ was preferred to $S_{1}$. Our sensitivity analysis of the importance weights showed that for each pair of scenarios, all calculated weight changes were infeasible. In other words, change of 
weight in $F_{1}, F_{2}, F_{3}$, or $F_{4}$ resulted in no rank reversal (see Tables 7.2 and 7.3). This was primarily because $S_{3}$ was preferred to $S_{2}$ and $S_{2}$ was preferred to $S_{1}$ on not only the overall weighted performance score but also on the performance score of each evaluation factor. Next, we performed a sensitivity analysis on the performance values associated with the four factors. Table 7.4 presents the percentage change of the performance scores that result in a rank reversal. For example, for a ranking reversal of $S_{1}$ and $S_{2}$, the performance scores of $S_{1}$ and $S_{2}$ would have to be increased by $26,14,14$, and $10 \%$, respectively.

Table 7 Sensitivity analysis results for $t+15$

Table 7.1 Decision matrix and preferences $\left(s_{x, r}\right.$ and $\left.F_{r}\right)$

\begin{tabular}{lccccc}
\hline & $F_{1}$ & $F_{2}$ & $F_{3}$ & $F_{4}$ & \\
\cline { 2 - 5 } Scenario & $w_{1}=0.100$ & $w_{2}=0.196$ & $w_{3}=0.404$ & $w_{4}=0.300$ & $F_{r}$ \\
\hline $\mathrm{S}_{1}$ & 0.769 & 0.750 & 0.354 & 0.667 & 0.567 \\
$\mathrm{~S}_{2}$ & 0.769 & 0.750 & 0.405 & 0.667 & 0.587 \\
$\mathrm{~S}_{3}$ & 0.769 & 0.750 & 0.426 & 0.667 & 0.596 \\
\hline
\end{tabular}

Table 7.2 All possible $\delta_{r, x, y}$ values (absolute change in criteria weights)

\begin{tabular}{lcccc}
\hline Scenario pairs & $F_{1}$ & $F_{2}$ & $F_{3}$ & $F_{4}$ \\
\hline$S_{1}, S_{2}$ & $\mathrm{NF}$ & $\mathrm{NF}$ & $\mathrm{NF}$ & $\mathrm{NF}$ \\
$S_{1}, S_{3}$ & $\mathrm{NF}$ & $\mathrm{NF}$ & $\mathrm{NF}$ & $\mathrm{NF}$ \\
$S_{2}, S_{3}$ & $\mathrm{NF}$ & $\mathrm{NF}$ & $\mathrm{NF}$ & $\mathrm{NF}$ \\
\hline
\end{tabular}

Table 7.3 All possible $\% w_{r, x, y}$ values (percent change in criteria weights)

\begin{tabular}{lcccc}
\hline Scenario pairs & $F_{1}$ & $F_{2}$ & $F_{3}$ & $F_{4}$ \\
\hline $\mathrm{S}_{1}, \mathrm{~S}_{2}$ & $\mathrm{NF}$ & $\mathrm{NF}$ & $\mathrm{NF}$ & $\mathrm{NF}$ \\
$\mathrm{S}_{1}, \mathrm{~S}_{3}$ & $\mathrm{NF}$ & $\mathrm{NF}$ & $\mathrm{NF}$ & $\mathrm{NF}$ \\
$\mathrm{S}_{2}, \mathrm{~S}_{3}$ & $\mathrm{NF}$ & $\mathrm{NF}$ & $\mathrm{NF}$ & $\mathrm{NF}$ \\
\hline
\end{tabular}

Table 7.4 All possible $\% R_{r, x, y}$ values (percent change in performance scores)

\begin{tabular}{lrrrr}
\hline Scenario pairs & $F_{1}$ & $F_{2}$ & $F_{3}$ & $F_{4}$ \\
\hline $\mathrm{S}_{1}, \mathrm{~S}_{2}$ & -26 & -14 & -14 & -10 \\
$\mathrm{~S}_{1}, \mathrm{~S}_{3}$ & -38 & -20 & -20 & -15 \\
$\mathrm{~S}_{2}, \mathrm{~S}_{1}$ & 26 & 14 & 12 & 10 \\
$\mathrm{~S}_{2}, \mathrm{~S}_{3}$ & -12 & -6 & -6 & -5 \\
$\mathrm{~S}_{3}, \mathrm{~S}_{1}$ & 38 & 20 & 17 & 15 \\
$\mathrm{~S}_{3}, \mathrm{~S}_{2}$ & 12 & 6 & 5 & 5 \\
\hline
\end{tabular}

Note: NF denotes non-feasible.

At $t+25$, we considered two scenarios, $S_{1}(t+25 \mathrm{~A})$ and $S_{2}(t+25 \mathrm{~B}) . S_{2}$ with an overall weighted performance score of 0.655 was preferred to $S_{1}$ with an overall weighted performance score of 0.507 (see Table 8.1). The results for our sensitivity analysis of the importance weights are presented in Tables 8.2 and 8.3. Changes for $F_{2}$ and $F_{3}$ were considered feasible and changes for $F_{1}$ and $F_{4}$ were considered infeasible. As shown in 
Table 8.2, the weight on $F_{2}$ would have to decrease by -2.911 (or increase of 2.911) in order for the ranking of $S_{1}$ and $S_{2}$ to reverse. Furthermore, the current 0.404 weight of $F_{3}$ would have to decrease by 0.388 for the ranking of $S_{1}$ and $S_{2}$ to reverse $(0.404-$ $0.388=0.016$ ). This information is presented in Table 8.3 in percentage terms. For example, the weight on $F_{2}$ would have to decrease by $-1456 \%$ (or an increase of 1456\%) or the weight on $F_{3}$ would have to decrease by $97 \%$ in order for the rankings to reverse. Finally, the sensitivity analysis on the performance scores of the two scenarios is presented in Table 8.4. For example, the performance score of $S_{1}$ on $F_{1}, 0.642$, would have to decrease by -227 (or an increase of 227\%) in order for the rankings of $S_{1}$ and $S_{2}$ to reverse.

Table 8 Sensitivity analysis results for $t+25$

Table 8.1 Decision matrix and preferences $\left(s_{x, r}\right.$ and $\left.F_{r}\right)$

\begin{tabular}{lccccc}
\hline & $F_{1}$ & $F_{2}$ & $F_{3}$ & $F_{4}$ & \\
\cline { 2 - 5 } Scenario & $w_{1}=0.100$ & $w_{2}=0.196$ & $w_{3}=0.404$ & $w_{4}=0.300$ & $F_{r}$ \\
\hline $\mathrm{S}_{1}$ & 0.642 & 1.000 & 0.117 & 0.667 & 0.507 \\
$\mathrm{~S}_{2}$ & 0.696 & 0.950 & 0.492 & 0.667 & 0.655 \\
\hline
\end{tabular}

Table 8.2 All possible $\delta_{r, x, y}$ values (absolute change in criteria weights)

\begin{tabular}{lcccr}
\hline Scenario pairs & $F_{1}$ & $F_{2}$ & $F_{3}$ & $F_{4}$ \\
\hline $\mathrm{S}_{1}, \mathrm{~S}_{2}$ & $\mathrm{NF}$ & -2.911 & 0.388 & $\mathrm{NF}$ \\
\hline
\end{tabular}

Table 8.3 All possible $\% w_{r, x, y}$ values (percent change in criteria weights)

\begin{tabular}{lcccc}
\hline Scenario pairs & $F_{1}$ & $F_{2}$ & $F_{3}$ & $F_{4}$ \\
\hline $\mathrm{S}_{1}, \mathrm{~S}_{2}$ & $\mathrm{NF}$ & -1456 & 97 & $\mathrm{NF}$ \\
\hline
\end{tabular}

Table 8.4 All possible $\% R_{r, x, y}$ values (percent change in performance scores)

\begin{tabular}{lrrrr}
\hline Scenario pairs & $F_{1}$ & $F_{2}$ & $F_{3}$ & $F_{4}$ \\
\hline $\mathrm{S}_{1}, \mathrm{~S}_{2}$ & -227 & -73 & -311 & -73 \\
$\mathrm{~S}_{2}, \mathrm{~S}_{1}$ & $\mathrm{NF}$ & 77 & 74 & 73 \\
\hline
\end{tabular}

Note: NF denotes non-feasible

\section{Conclusions and future research directions}

The existing vehicle-target assignment models are either static, lack flexibility, single objective, or lack capabilities to consider the value of connectivity in a networked environment. In contrast, the model presented in this study is:

1 dynamic, since the outcome of previous engagements is used in making future assignments

2 flexible, since it allows multiple roles for the vehicles 
3 multi-objective, since it considers a series of competing objectives simultaneously

4 communicative, since it takes into account the value of connectivity and communication.

We utilise real-time information via a robust network to enhance information sharing, collaboration, shared situational awareness, and self-synchronisation.

The MCDA model presented in this study is a solution evaluation tool that can be embedded within genetic algorithms, simulated annealing, or Tabu search environments. Aside from its applicability to vehicle-target allocation, the model has wider applications in MCDA situations where multiple roles are dynamically assigned to multiple resources. For example, in disaster recovery management, varying roles such as rescue operations, law enforcement, and communication facilitation are assigned to various personnel in response to emerging events. We are currently working on a dynamic optimisation model that incorporates the multi-criteria objectives of maximising the value of destroyed targets, minimising the threat to vehicles, and incorporates the value of communication. We are also considering embedding the evaluation model presented in this study along with our optimisation model into a visualisation environment within a larger decision support system.

\section{Acknowledgements}

This research was supported by the US Air Force Research Laboratory grant numbers FA9550-04-C-0141 and FA8750-05-2-0064. Approved for public release.

\section{References}

Barron, F.H. and Barnett, B.E. (1996) 'Decision quality using ranked attribute weights', Management Science, Vol. 42, pp.1515-1523.

Beard, R.W., McLain, T.W. and Goodrich, M.A. (2002) 'Coordinated target assignment and intercept for unmanned air vehicles', IEEE Transaction on Robotics and Automation, Vol. 18, pp.911-922.

Braford, J.C. (1961) 'Determination of optimal assignment of a weapon system to several targets', AEREITM-9, Vought Aeronautics, Dallas, TX.

Castanon, D.A. (1987) 'Advanced weapon-target assignment algorithm quarterly report', TR-337, ALPHATECH Inc., Burlington, MA.

Chang, S.C., James, R.M. and Shaw, J.J. (1987) 'Assignment algorithm for kinetic energy weapons in boost defense', Paper presented in the Proceedings of the IEEE 26th Conference on Decision and Control, Los Angeles, CA.

Day, R.H. (1966) 'Allocating weapons to target complexes by means of nonlinear programming', Operations Research, Vol. 14, pp.992-1013.

DenBroeder, G.G. Jr., Ellison, R.E. and Emerling, L. (1959) 'On optimal target assignments', Operations Research, Vol. 7, pp.322-326.

Dyer, J.S. (1990a) 'Remarks on the analytic hierarchy process', Management Science, Vol. 36, pp.249-258.

Dyer, J.S. (1990b) 'A clarification of 'remarks on the analytic hierarchy process', Management Science, Vol. 36, pp.274-275.

Eckler, A.R. and Burr, S.A. (1972) Mathematical Models of Target Coverage and Missile Allocation. Alexandria, VA: Military Operations Research Society. 
Edwards, W. and Barron, F.H. (1994) 'SMART and SMARTER: improved simple methods for multiattribute utility measurement', Organizational Behavior and Human Decision Processes, Vol. 60, pp.306-325.

Expert Choice (2004) Computer Software. McLean, VA: Decision Support Software, Inc.

Flint, M. (2003) 'Stochastic models of cooperative autonomous UAV search problem', Military Operations Research, Vol. 8, pp.13-32.

Forman, E. and Gass, S.I. (2001) 'The analytic hierarchy process: an exposition', Operations Research, Vol. 49, pp.469-486.

Gass, S.I. (2005) 'Model world: the great debate - MAUT versus AHP', Interfaces, Vol. 35, pp.308-312.

Glover, F. and Laguna, M. (1996) Tabu Search. Dordrecht, The Netherlands: Kluwer Academic Publishers.

Grant, K.E. (1993) 'Optimal resource allocation using genetic algorithms', Naval Review (pp.174-175). Washington, DC: Naval Research Laboratory.

Green, D.J., Moore, J.T. and Borsi, J.J. (1997) 'An integer solution heuristic for the arsenal exchange model (AEM)', Military Operations Research, Vol. 3, pp.5-15.

Goldberg, D.E. (1989) Genetic Algorithms in Search, Optimization, and Machine Learning. Reading, MA: Addison-Wesley.

Golden, B. and Wasil, E. (2003) 'Celebrating 25 years of AHP-based decision making’, Computers and Operations Research, Vol. 30, pp.1419-1497.

Golden, B., Wasil, E. and Harker, P. (1989) The Analytic Hierarchy Process: Applications and Studies. Berlin, Germany: Springer.

Harker, P.T. and Vargas, L.G. (1987) 'The theory of ratio scale estimation: Saaty's analytic hierarchy process’, Management Science, Vol. 33, pp.1383-1403.

Harker, P.T. and Vargas, L.G. (1990) 'Reply to 'Remarks on the analytic hierarchy process' by J.S. Dyer’, Management Science, Vol. 36, pp.269-273.

Holland, J. (1975) Adaptation in Natural and Artificial Systems. Ann Arbor, MI: University of Michigan Press.

Hosein, P., Walton, J. and Athans, M. (1988) 'Dynamic weapon-target assignment problems with vulnerable C3 nodes', Paper presented in the Proceedings of the 1988 Command and Control Symposium.

Katter, J.D. (1986) 'A solution of the multi-weapon, multi-target assignment problem', Working paper 26957, MITRE.

Lee, Z-J. and Lee, C-Y. (2003) 'A hybrid search algorithm of ant colony optimization and genetic algorithm applied to weapon-target assignment problems', in J. Liu, Y. Cheung and H. Yin (Eds), Intelligent Data Engineering and Automated Learning (pp.278-285). New York, NY: Springer.

Lee, Z-J. and Lee, C-Y. (2005) 'A hybrid search algorithm with heuristics for resource allocation problem', Informatics and Computer Science, Vol. 173, pp.155-167.

Lee, Z-J., Lee, S-G. and Lee, C-Y. (2003) 'Efficiently solving general weapon-target assignment problem by genetic algorithms with greedy eugenics', IEEE Transactions on Systems Man and Cybernetics Part B, Vol. 33, pp.113-121.

Li, V.C., Curry, G.L. and Boyd, E.A. (2004) 'Towards the real time solution of strike force asset allocation problems', Computers and Operations Research, Vol. 31, pp.273-291.

Manne, A.S. (1958) ‘A target assignment problem’, Operations Research, Vol. 6, pp.346-351.

Matlin, S.M. (1970) 'A review of the literature on the missile-allocation problem', Operations Research, Vol. 18, pp.334-373.

Metler, W.A. and Preston, F.L. (1990) 'A suite of weapon assignment algorithms for a SDI mid-course battle manager’, NRL Memorandum Report 671. Washington, DC: Naval Research Laboratory. 
Miller, L.E. (2001) Multihop Connectivity of Arbitrary Networks. Available at: http://w3.antd.nist.gov/wctg/netanal/ConCalc.pdf.

Murphey, R.A. (1999) 'Target-based weapon target assignment problems', P.M. Pardalos and L.S. Pitsoulis (Eds), Nonlinear Assignment Problems: Algorithms and Applications (Vol. 7, pp.39-53). Dordrecht, The Netherlands: Kluwer Academic Publishers.

Orlin, D. (1987) 'Optimal weapons allocation against layered defenses', Naval Research Logistics, Vol. 34, pp.605-616.

O’Rourke, K.P., Bailey, T.G., Hill, R. and Carlton, W.B. (2000) 'Dynamic routing of unmanned aerial vehicles using reactive Tabu Search’, Military Operations Research, Vol. 6, pp.33-42.

Reeves, C.R. (1993) Modern Heuristic Techniques for Combinatorial Problems. New York, NY: John Wiley and Sons.

Saaty, T.L. (1977) 'A scaling method for priorities in hierarchical structures', Journal of Mathematical Psychology, Vol. 15, pp.234-281.

Saaty, T.L. (1983) 'Axiomatic foundations of the analytic hierarchy process', Management Science, Vol. 32, pp.841-855.

Saaty, T.L. (1990) 'An exposition of the AHP in reply to the paper 'Remarks on the analytic hierarchy process’', Management Science, Vol. 36, pp.259-268.

Saaty, T.L. (1994) 'How to make a decision: the analytic hierarchy process', Interfaces, Vol. 24, pp.19-43.

Shannon, C. (1948) 'A mathematical theory of communication', The Bell System Technical Journal, Vol. 27, pp.379-423.

Shim, J.P. (1989) 'Bibliographical research on the Analytic Hierarchy Process (AHP)', Socio-Economic Planning Sciences, Vol. 23, pp.161-67.

Solymosi, T. and Dombi, J. (1986) 'A method for determining weights of criteria: the centralized weights', European Journal of Operational Research, Vol. 26, pp.35-41.

Toth, P. and Vigo, D. (2002) The Vehicle Routing Problem. Philadelphia, PA: SIAM.

Triantaphyllou, E. (2000) Multi-Criteria Decision Making Methods: A Comparative Study. Boston, MA: Kluwer Academic Publishers.

Triantaphyllou, E. and Sanchez, A. (1997) 'A sensitivity analysis approach for some deterministic multi-criteria decision-making methods’, Decision Sciences, Vol. 28, pp.151-194.

Van Laarhoven, P.J.M. and Aarts, E. (1987) Simulated Annealing: Theory and Applications. Dordrecht, The Netherlands: D. Reidel Publishing.

Von Winterfeldt, D. and Edwards, W. (1986) Decision Analysis and Behavioral Research. Cambridge, UK: Cambridge University Press.

Voss, S. (1999) 'Heuristics for nonlinear assignment problems', in P.M. Pardalos and L.S. Pitsoulis (Eds), Nonlinear Assignment Problems: Algorithms and Applications (Vol. 7, pp.175-215). Boston, MA: Kluwer Academic Publishers.

Wachholder, E. (1989) 'A neural network-based optimization algorithm for the static weapontarget assignment problem’, ORSA Journal on Computing, Vol. 4, pp.232-246.

Weiss, E.N. and Rao, V.R. (1987) 'AHP design issues for large-scale systems’, Decision Sciences, Vol. 18, pp.43-61.

Zahedi, F. (1986) 'The analytical hierarchy process - a survey of the method and its applications', Interfaces, Vol. 16, pp.96-108. 